\title{
The prospective application of a hypoxic radiosensitizer, doranidazole to rat intracranial glioblastoma with blood brain barrier disruption
}

\author{
Hironobu Yasui ${ }^{1}$, Taketoshi Asanuma ${ }^{2}$, Junichi Kino ${ }^{1}$, Tohru Yamamori ${ }^{1}$, Shunsuke Meike ${ }^{1}$, Masaki Nagane ${ }^{1}$ \\ Nobuo Kubota ${ }^{3}$, Mikinori Kuwabara ${ }^{1}$ and Osamu Inanami ${ }^{1 *}$
}

\begin{abstract}
Background: Glioblastoma is one of the intractable cancers and is highly resistant to ionizing radiation. This radioresistance is partly due to the presence of a hypoxic region which is widely found in advanced malignant gliomas. In the present study, we evaluated the effectiveness of the hypoxic cell sensitizer doranidazole (PR-350) using the C6 rat glioblastoma model, focusing on the status of blood brain barrier (BBB).

Methods: Reproductive cell death in the rat C6 glioma cell line was determined by means of clonogenic assay. An intracranial C6 glioma model was established for the in vivo experiments. To investigate the status of the BBB in C6 glioma bearing brain, we performed the Evans blue extravasation test. Autoradiography with $\left[{ }^{14} \mathrm{C}\right]$-doranidazole was performed to examine the distribution of doranidazole in the glioma tumor. T2-weighted MRI was employed to examine the effects of X-irradiation and/or doranidazole on tumor growth.

Results: Doranidazole significantly enhanced radiation-induced reproductive cell death in vitro under hypoxia, but not under normoxia. The BBB in C6-bearing brain was completely disrupted and $\left[{ }^{14} \mathrm{C}\right]$-doranidazole specifically penetrated the tumor regions. Combined treatment with X-irradiation and doranidazole significantly inhibited the growth of C6 gliomas.

Conclusions: Our results revealed that BBB disruption in glioma enables BBB-impermeable radiosensitizers to penetrate and distribute in the target region. This study is the first to propose that in malignant glioma the administration of hydrophilic hypoxic radiosensitizers could be a potent strategy for improving the clinical outcome of radiotherapy without side effects.
\end{abstract}

Keywords: Doranidazole, Radiosensitizer, Glioblastoma, Hypoxia

\section{Background}

Glioblastoma, a highly malignant brain tumor, usually has a poor prognosis despite surgical treatment, radiation therapy and/or chemotherapy [1,2]. Even when recognizable tumor mass can be surgically removed and adjuvant radiotherapy and chemotherapy are employed, the mean survival of patients is only extended from 2-3 months to 1 year [3]. Several factors are considered to be responsible for the radioresistance of glioblastomas

\footnotetext{
* Correspondence: inanami@vetmed.hokudai.ac.jp

1 Laboratory of Radiation Biology, Department of Environmental Veterinary Sciences, Graduate School of Veterinary Medicine, Hokkaido University, Kita 18 Nishi 9, Kita-ku, Sapporo, Hokkaido, Japan

Full list of author information is available at the end of the article
}

such as hypoxia [4], the up-regulation of the EGFR pathway [5] and the existence of glioma stem cells [6]. Tumor hypoxia, which is generally attributed to the imbalance between the demand and supply of oxygen and poorly organized vasculature $[7,8]$, is observed in many tumor types especially glioblastoma. Hypoxia appears to be the most important factor in the development of radioresistance, invasiveness and more aggressive tumor phenotypes [9]. Therefore, to develop therapies against glioblastoma, an invariably fatal disease, enhancement of the efficacy of radiotherapy by means of hypoxic radiosensitizers is certainly a promising way to achieve improved therapeutic outcome.

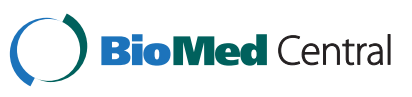


Numerous radiosensitizers for hypoxic cells have been developed and screened, both in preclinical studies and clinical trials $[10,11]$. The nitroimidazole derivatives are major compounds in this regard and have been tested extensively. However, most clinical trials have failed to demonstrate significant efficacy using these sensitizers, mainly because of undesirable side effects such as neurotoxicity [12]. However, clinical trials in Denmark reported that misonidazole and nimorazole were effective in chemoradiotherapy against carcinomas of the larynx and pharynx $[13,14]$. The efficacy of nitroimidazole derivatives as hypoxic radiosensitizers remains controversial. It is currently difficult to determine which type of tumor is susceptible to hypoxic radiosensitization and which regimen is most efficient using nonproprietary drugs, because of the lack of financial incentives for the pharmaceutical industries to evaluate them [11].

Doranidazole (1-[1,3',4'-trihydroxy-2'-butoxy]-methyl2-nitroimidazole [PR-350]) is a hypoxic radiosensitizer, and is a derivative of 2-nitroimidazole intended to reduce neurotoxicity due to its blood brain barrier (BBB) impermeability $[15,16]$. Several studies have shown that doranidazole has a radiosensitizing effect under hypoxia, both in vitro [17-19] and in vivo [19-21]. Based on these studies, a phase III trial of doranidazole against advanced pancreatic cancer was performed; it was demonstrated that treatment with doranidazole following radiation significantly improved the tumor mass reduction rate and extended patient survival [22]. While various results have suggested that doranidazole has promising potential in hypoxia-targeting chemoradiotherapy, to date there have not been any reports on the use of this drug for intracranial glioma.

It is known that the $\mathrm{BBB}$ restricts the transport of hydrophilic or high-molecular-weight compounds into the brain to maintain the brain internal milieu. Therefore, doranidazole, which has a hydrophilic residue, cannot cross the $\mathrm{BBB}$ and cause any toxicity to the intact brain. However, in many advanced malignant gliomas, disruption of the BBB has been reported [23-25]. These facts led us to consider the possibility that doranidazole might only reach the tumor regions and not the surrounding healthy brain.

In the present study, we examined the radiosensitizing effect of doranidazole on C6 glioma both in vitro and in vivo. We particularly focused on the extent of $\mathrm{BBB}$ disruption in C6-bearing rat brain and also investigated the uptake of doranidazole in the tumor region.

\section{Methods}

\section{Materials}

Doranidazole and 2 '- $\left[{ }^{14} \mathrm{C}\right]$-labeled doranidazole $\left(\left[{ }^{14} \mathrm{C}\right]\right.$ doranidazole) were supplied by POLA PHARMA INC. (Tokyo, Japan). The Hypoxyprobe ${ }^{\mathrm{TM}}-1$ Kit was obtained from Hypoxyprobe Inc. (Burlington, MA, USA). A BD Matrigel $^{\mathrm{TM}}$ reagent was purchased from BD Biosciences (Billerica, MA, USA). Ultrapure $\mathrm{N}_{2}$ gas (99.999\%) was obtained from Air Water Technical Supply (Ishikari, Japan). Other chemicals were purchased from Wako Pure Chemical Industries, Ltd. (Tokyo, Japan) unless otherwise stated.

\section{Cell culture}

Rat glioma cell line C6 was obtained from the Health Science Research Resources Bank (Osaka, Japan). The cells were maintained in Dulbecco's modified Eagle's medium (DMEM; Gibco-BRL/Invitrogen, Carlsbad, CA, USA) supplemented with 10\% fetal bovine serum (FBS: Filtron, Brooklyn, Australia) at $37^{\circ} \mathrm{C}$ in $5 \% \mathrm{CO}_{2} / 95 \%$ air.

\section{Cell incubation, $\mathrm{X}$-irradiation and drug treatment in vitro}

Tumor cells attached to a $6-\mathrm{cm}$ plastic dish were treated with $10 \mathrm{mM}$ doranidazole before hypoxic incubation. The hypoxic condition (oxygen concentration $\leq 10$ $\mathrm{mmHg}$ [1.3\%]; unpublished data) for tumor cells in the dish was achieved by placing it in a gas-exchangeable chamber [18] and continuously passing ultrapure $\mathrm{N}_{2}$ gas for 25 minutes on ice. The cells were then exposed to 20 Gy of X-rays while maintaining the gas flow. Xirradiation was performed with a Shimadzu PANTAK HF-350 X-ray generator (1.0 mm Al filter; $200 \mathrm{kVp}$; 20 mA; Shimadzu, Kyoto, Japan).

\section{Clonogenic survival assay}

After X-irradiation under hypoxia or normoxia, C6 cells were collected by trypsinization and washed with PBS. The proper number (200-30000) of cells were seeded on a $6-\mathrm{cm}$ plastic dish containing fresh medium with $10 \%$ fetal bovine serum, followed by incubation at $37^{\circ} \mathrm{C}$ for $8-$ days. The cells were then fixed with methanol, stained with Giemsa solution and scored under a microscope. Only colonies containing more than 50 cells were scored as surviving cells. The surviving fraction at each dose was calculated with respect to the plating efficiency of the nonirradiated control.

\section{Animals}

WKAH/Hkm rats aged 9 weeks were purchased from Japan SLC (Hamamatsu, Japan). All animal experiments in this study were conducted according to the guidelines of the Law for The Care and Welfare of Animals in Japan and approved by the Animal Experiment Committee of the Graduate School of Veterinary Medicine, Hokkaido University.

\section{Intracranial tumor model}

The C6 intracranial tumor model was established according to the method detailed in our previous study 
[26]. Anesthetized rats were placed on a stereotaxic device (Narishige Scientific Instrument Lab., Tokyo, Japan). A 1-mm hole was drilled through the skull $2 \mathrm{~mm}$ anterior and $2 \mathrm{~mm}$ lateral to the bregma on the right-hand side of the head. One million of C6 cells in a mixture of $5 \mu \mathrm{L}$ FBS(-) culture media and $5 \mu \mathrm{L}$ Matrigel were injected into the cortex at a 3-mm depth at a rate of $2 \mu \mathrm{L} / \mathrm{min}$. A waiting time of 2 minutes was implemented following injection and the hole was closed using bone wax. The incision was sutured and covered with surgical glue.

\section{Evaluation of the BBB disruption in C6-bearing rats}

Vascular permeability in C6-bearing brain was evaluated by perfusing it with Evans blue dye according to the method described previously [27]. In brief, Evans blue dye solution (2\%) was intravenously administered to rats at a dose of $3 \mathrm{ml} / \mathrm{kg}$ and allowed to circulate for 60 minutes. To remove intravascular dye, rats were transcardially perfused with saline for 20 minutes. Brains were removed and sectioned at a thickness of $2 \mathrm{~mm}$.

\section{Treatment with doranidazole and X-irradiation}

Doranidazole administration and X-irradiation were performed when the tumor reached a size of 50$100 \mathrm{~mm}^{3}$. Animals were randomized into four groups: (1) no treatment; (2) X-irradiation (6 Gy) alone; (3) doranidazole administration alone; and (4) doranidazole administration at 30 minutes before X-irradiation (6 Gy). Doranidazole at a dose of $200 \mathrm{mg} / \mathrm{kg}$ was intravenously

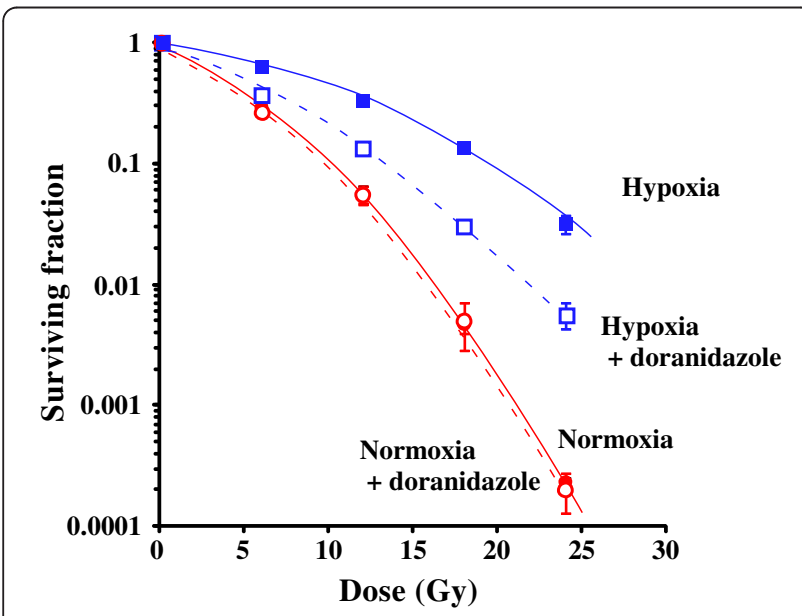

Figure 1 Sensitization of $\mathrm{C} 6$ cells to radiation under hypoxia using doranidazole. Dose-response curves of X-irradiated C6 cells. Tumor cells were X-irradiated under normoxia (red closed circles), under normoxia with doranidazole (red open circles), under hypoxia (blue closed squares) and under hypoxia with doranidazole (blue open squares). The surviving fraction at each dose was calculated and corrected according to the plating efficiency of the

nonirradiated control. Data are expressed as the mean \pm S.E. for three experiments. (i.v.) injected into rats. For irradiation of intracranial tumors, rats were shielded with lead panels, except for the tumor-bearing cranium. X-irradiation was performed with a Shimadzu PANTAK HF-350 X-ray generator at a dose rate of $1.2 \mathrm{~Gy} / \mathrm{min}$.

\section{MRI experiments}

MRI was carried out using a $7.05 \mathrm{~T}$ superconducting magnet (Oxford Instruments, Oxford, UK) equipped with a Unity/Inova 300/183 spectrometer (Varian, Palo Alto, CA, USA). Rats were placed in the center of a $35 \mathrm{~mm}$ diameter quadrature RF coil. After rapid assessment of the tumor position using a multislice spin-echo (MSE) sequence, T2weighted images (T2WIs) were also obtained using a MSE sequence with $\mathrm{TR} / \mathrm{TE}=2000 \mathrm{~ms} / 60 \mathrm{~ms}, \mathrm{FOV}=80 \times 80$ and $60 \times 60 \mathrm{~mm}$ (for sagittal and coronal images, respectively), image matrix $=128 \times 128$ and slice thickness = $1 \mathrm{~mm}$. Using lengths of tumors measured in three orthogonal dimensions, tumor volume $(V)$ was calculated as: $V$ $\left(\mathrm{mm}^{3}\right)=\pi(a \times b \times c) / 6$, where $a, b$ and $c$ represent width, height and thickness, respectively.

To measure leakage from the $\mathrm{BBB}$, a gadolinium-chelate (Gd-[DTPA]) contrast material (Magnevist ${ }^{\circledR}$, gadopentetate dimeglumine: Bayer Healthcare Pharmaceuticals, Montville, NJ, USA) was i.v. injected at a concentration of $0.1 \mathrm{mmol} /$ $\mathrm{kg}$ body weight. Contrast-enhanced MRI (CE-MRI) images were obtained using multislice T1-weighted images (T1WIs) with spin-echo sequences. The parameters of the CE-MRI were $\mathrm{TR} / \mathrm{TE}=500 \mathrm{~ms} / 16 \mathrm{~ms}$, slice thickness = $1 \mathrm{~mm}, \mathrm{FOV}=51.2 \times 51.2 \mathrm{~mm}$, and image matrix $=256 \times$ 256. The quantification of the signal enhancement due to Gd-[DTPA] uptake to glioma was performed using Image J software (National Institutes of Health, Bethesda, MD, USA) by calculating the ratio of signal intensity in tumor region to that in normal brain region.

\section{Autoradiography}

To examine the distribution of doranidazole in the rat brain, we performed autoradiographic analysis using $\left[{ }^{14} \mathrm{C}\right]$-doranidazole. Tumor-bearing rats were i.v. injected with $500 \mu \mathrm{L}$ of $\left[{ }^{14} \mathrm{C}\right]$-doranidazole $(4.9 \mathrm{MBq} /$ head). At 90 minutes after drug administration, rats were decapitated without prior perfusion with saline. Their brains were immediately removed and frozen. Frozen sections that were $20-\mu \mathrm{m}$ thick were exposed to a radiosensitive imaging plate (BAS-SR2040: Fuji Film Co. Ltd., Tokyo, Japan) for 4 days with a radioactive standard slide (ARC146: American Radiolabeled Chemicals Inc., St Louis, MO, USA). The image acquisition was performed using a BAS-2500 Bioimage Analyzer system (Fuji Film Co. Ltd. Tokyo, Japan). After the acquisition of autoradiographic images, parts of sections were fixed with $4 \%$ buffered formaldehyde and stained with hematoxylin/ $\operatorname{eosin}(\mathrm{H} / \mathrm{E})$. 
A
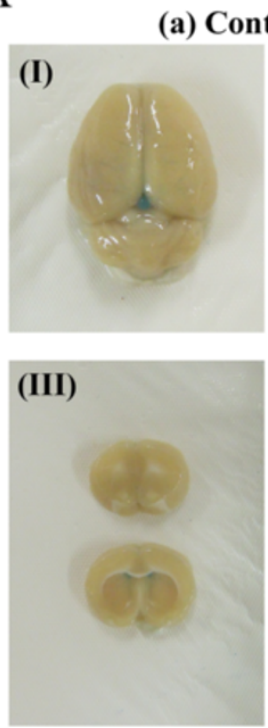

B

Before Gd-[DTPA] injection After Gd-[DTPA] injection
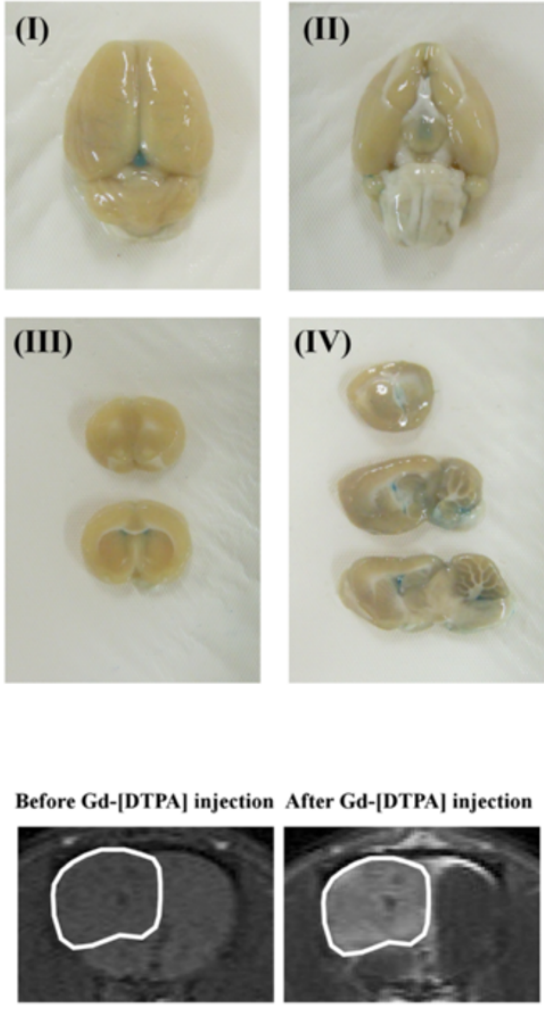

\section{(IV)}
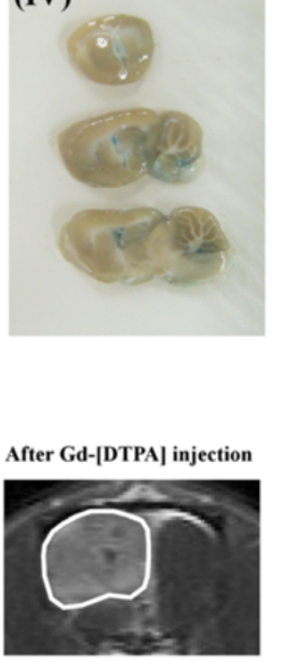

C

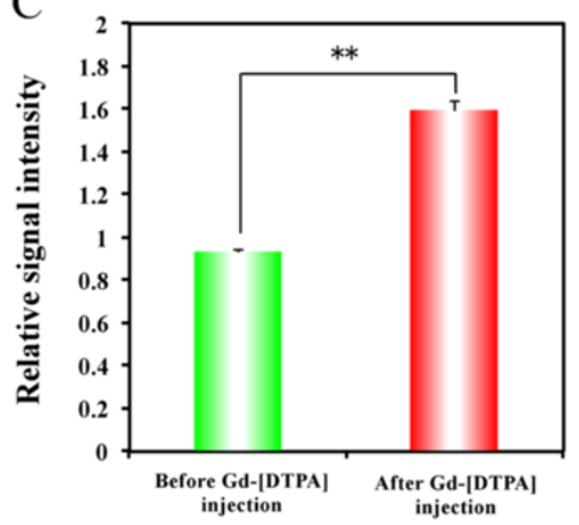

\section{(b) C6-bearing brain}
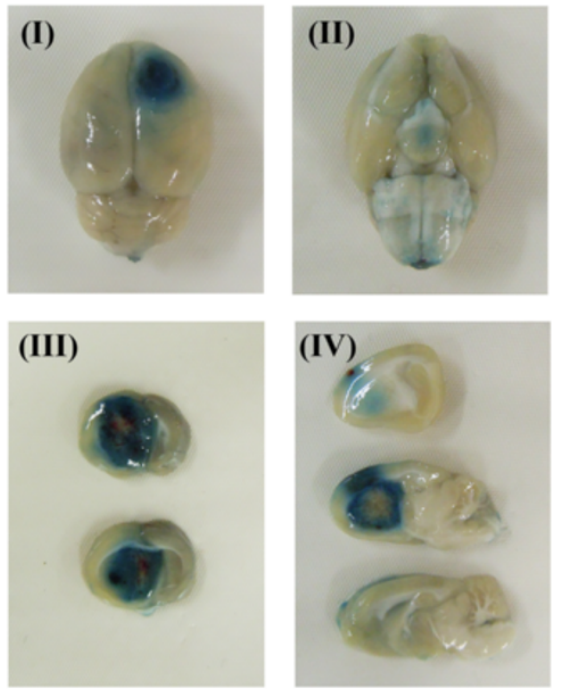


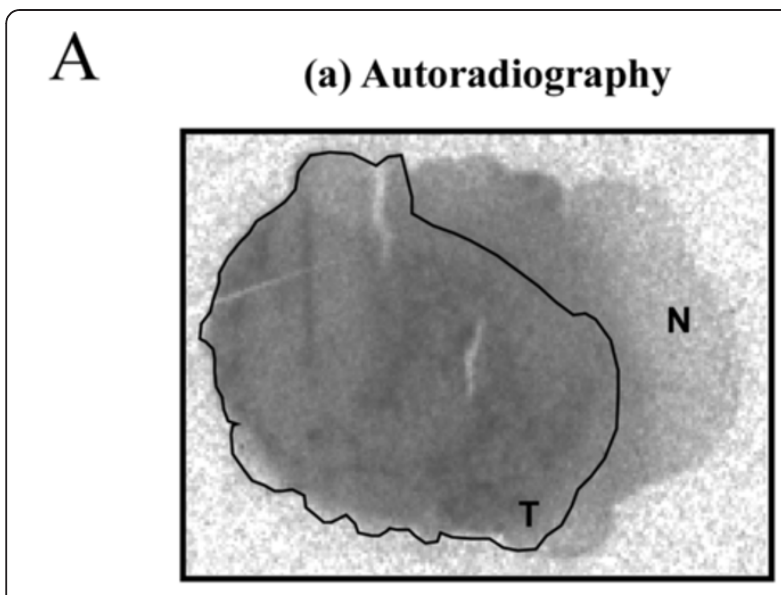

(b) $\mathbf{H} / \mathbf{E}$

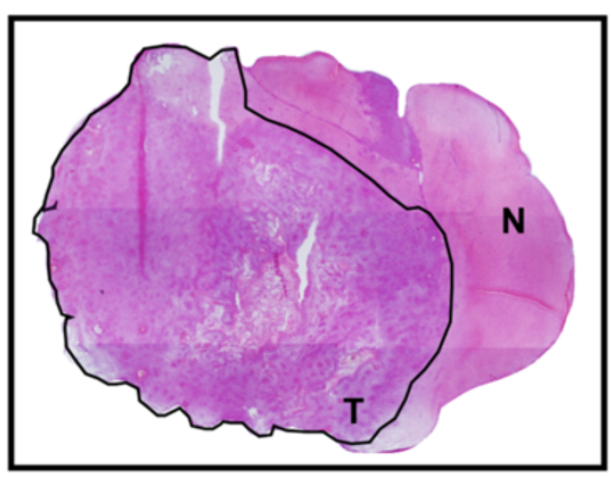

\section{B}

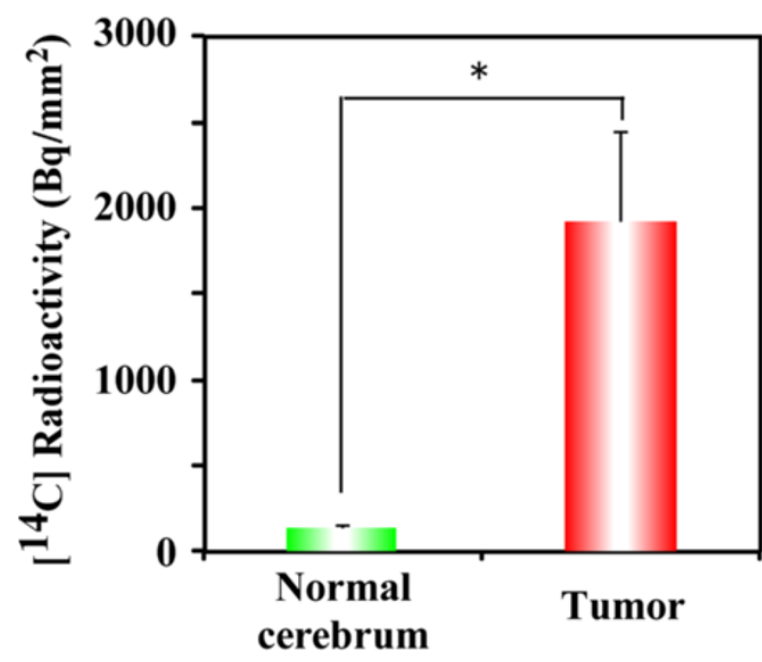

Figure 3 The distribution of $\left[{ }^{14} \mathrm{C}\right]$-doranidazole in $\mathrm{C} 6$ intracranial glioma. (A) A 20- $\mu \mathrm{m}$ thick tissue section of rat brain that was used for autoradiography (a) and subsequent $\mathrm{H} /$ E staining (b). Black lines show the C6 glioma. The annotated words "T" and "N" represent tumor and normal brain regions, respectively. (B) Using these images, quantitative data for the accumulation of $\left[{ }^{14} \mathrm{C}\right]$-doranidazole in normal cortex and C6 glioma was acquired. Data are expressed as the mean \pm S.E. for four different tumors. * $\mathrm{P}<0.05$ vs. normal cerebrum. were also stained with $\mathrm{H} / \mathrm{E}$. The stained images of each section were acquired using a fluorescence microscope (BZ-9000: Keyence, Osaka, Japan).

\section{Statistical analysis}

All results were expressed as the mean \pm S.E. The variance ratio was estimated using the F-test and differences in means of groups were determined using Student's ttest or Welch's t-test. The minimum level of significance was set at $\mathrm{P}<0.05$.

\section{Results}

The clonogenic survival curves for C6 glioma cells irradiated in vitro under normoxic and hypoxic conditions, with or without doranidazole, are shown in Figure 1. Under conditions without doranidazole, X-irradiation under hypoxia reduced the radiosensitivity of C6 cells, and the oxygen enhancement ratio (OER) was approximately 1.9. The hypoxic condition set in this experiment was $\leq 10 \mathrm{mmHg}$ for $\mathrm{pO}_{2}$, and this OER value coincided with that reported in a previous study [28]. Under normoxic conditions without irradiation, the survival fractions with or without doranidazole were $0.703 \pm$ 0.019 and $0.677 \pm 0.031$, respectively. Hypoxic conditions decreased the plating efficiency of C6 cells to $0.675 \pm$ 0.006 and the addition of doranidazole resulted in a further decline to $0.667 \pm 0.032$, although no significant differences were observed among the groups. Under both normoxia and hypoxia without irradiation, the toxicity of $10 \mathrm{mM}$ doranidazole against C6 cells was less than $30 \%$. While doranidazole had no sensitizing effect when combined with aerobic irradiation, it had significant sensitizing activity when combined with irradiation under hypoxic conditions. The dose that reduces cell survival to $10 \%\left(D_{10}\right)$ obtained from the hypoxic cell survival curve was $20.2 \mathrm{~Gy}$, and it decreased to $13.3 \mathrm{~Gy}$ when cells were irradiated in the presence of $10 \mathrm{mM}$ doranidazole. The sensitizing enhancement ratio (SER) for doranidazole after irradiation under hypoxic conditions was $\sim 1.5$, whereas the SER after irradiation under normoxic conditions was $\sim 1.0$.

To examine the disruption of the $\mathrm{BBB}$ in the C6tumor-bearing rat brain, we employed the Evans blue extravasation method. Evans blue dye is known to bind to albumin producing a $68 \mathrm{kDa}$ compound that does not cross the $\mathrm{BBB}$ [29]. In fact, normal control brain after intra-arterial infusion of Evans blue showed no staining in the cerebral hemisphere (Figure 2A [a-I, II]). Using this Evans blue extravasation test, we evaluated the permeability of the BBB in C6-bearing brain. Figure 2A (b-I, II) shows a clearly stained region in the frontal cortex of right hemisphere, in which the C6 tumor was located. The photographs in Figure 2A (III, IV) are views of sectioned slices from control and C6-bearing brains. They 
also demonstrated the apparent correspondence of the stained region with the tumor region in C6-bearing brain, while no staining was observed in the control brain. To confirm this disruption of the BBB in the tumor region, we performed CE-MRI analysis using a BBB-impermeable reagent, Gd-[DTPA]. Figure 2B displays representative pre- and post-contrast T1WIs of brains in C6-glioma-bearing rats, with the region of interest (ROI) placed on the glioma. After Gd-[DTPA]

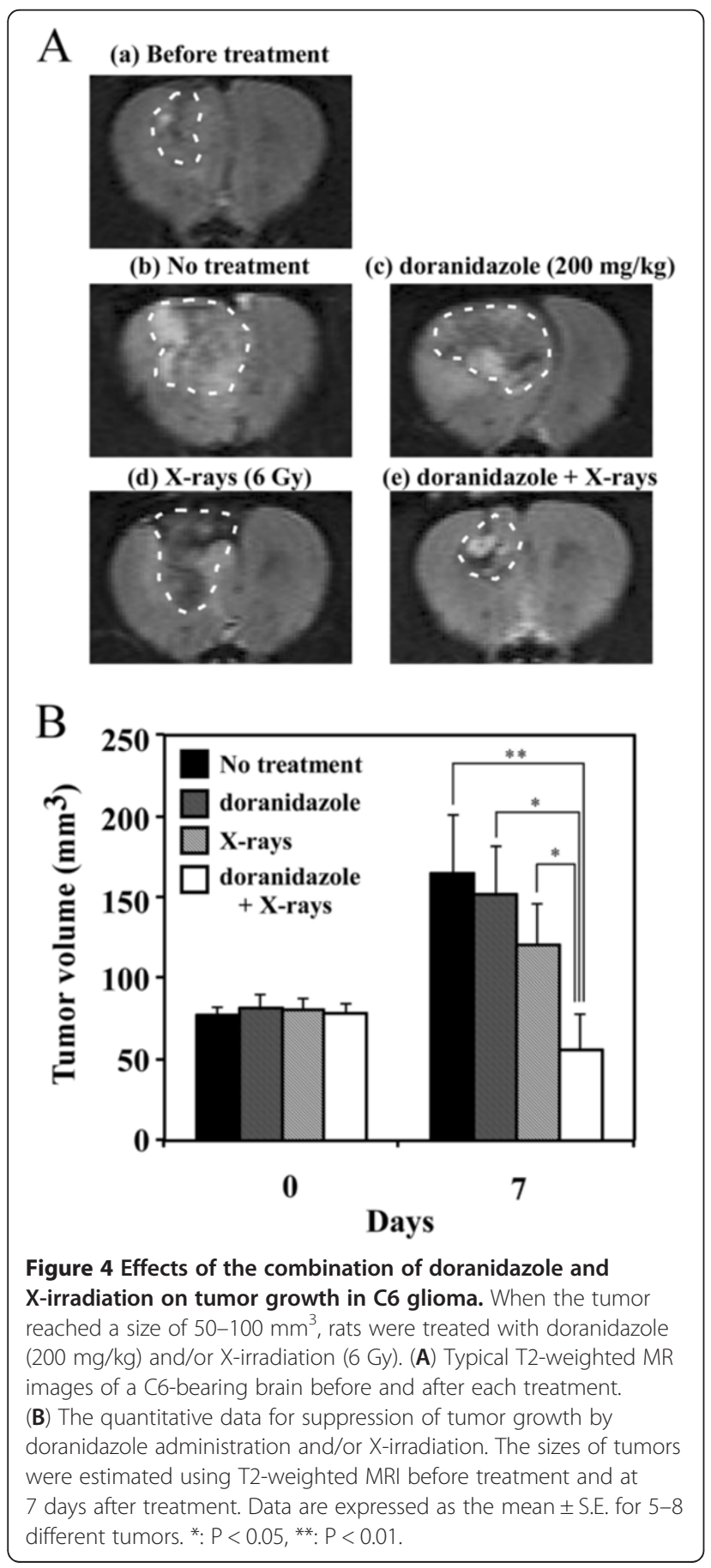

injection, MRI signal enhancement due to the accumulation of Gd-[DTPA] was clearly observed around the tumor region. The quantitative data showed that the relative signal intensities in glioma before and after Gd[DTPA] injection were $0.933 \pm 0.008$ and $1.597 \pm 0.042$, respectively (Figure $2 \mathrm{C}$ ).

We next investigated the distribution of doranidazole in the brains of C6-bearing rats. Ninety minutes after the i.v. administration of $\left[{ }^{14} \mathrm{C}\right]$-doranidazole, rats were decapitated. Brain tissue sections were analyzed using autoradiography and subsequent $\mathrm{H} / \mathrm{E}$ staining. In the autoradiographic image shown in Figure $3 \mathrm{~A}(\mathrm{a}),\left[{ }^{14} \mathrm{C}\right]-$ doranidazole is clearly distributed in the tumor region but not in the normal brain cortex. We then quantified the accumulation of $\left[{ }^{14} \mathrm{C}\right]$-doranidazole in each region of the normal cortex and tumor region defined by $\mathrm{H} / \mathrm{E}$ staining (Figure $3 \mathrm{~A}[\mathrm{~b}]$ ). Tumor regions showed significantly higher $\left[{ }^{14} \mathrm{C}\right]$ radioactivity levels (1926.5 \pm $\left.523.3 \mathrm{~Bq} / \mathrm{mm}^{2}\right)$ than the normal cortex region (138.7 \pm $14.6 \mathrm{~Bq} / \mathrm{mm}^{2}$ ) (Figure 3B). These results suggested that doranidazole could penetrate into the tumor region due to the breakdown of the BBB in the C6-bearing brain.

We also examined the radiosensitizing effect of doranidazole on the growth of transplanted C6 glioma. Rats with $50-100 \mathrm{~mm}^{3}$ of glioma tumor were treated with $200 \mathrm{mg} / \mathrm{kg}$ doranidazole and/or 6 Gy of X-rays. We estimated the tumor volumes before and after each treatment using T2WIs to indicate the definite tumor area (Figure 4A). As shown in Figure 4B, without any treatment tumor size increased $\sim 2.5$-fold in 7 days and reached $165.3 \pm$ $35.5 \mathrm{~mm}^{3}$. X-irradiation or doranidazole alone induced no statistically significant inhibition of tumor growth. The tumor volumes at 7 days after treatment were $121.0 \pm$ $24.9 \mathrm{~mm}^{3}$ after X-irradiation alone and $152.0 \pm 30.3 \mathrm{~mm}^{3}$ after doranidazole alone. X-irradiation at 30 minutes after doranidazole treatment induced a significant retardation in tumor growth $\left(56.0 \pm 22.7 \mathrm{~mm}^{3}\right)$. To examine the suppressive effect of doranidazole on the hypoxic region in the C6 glioma, histological analysis with pimonidazole staining and $\mathrm{H} / \mathrm{E}$ staining was performed. Immunohistological images for pimonidazole revealed a characteristic cord-like structure of hypoxia in viable tumor, within specimens resected from tumors receiving radiation or doranidazole alone. However, the great majority of the tumor containing hypoxic region was necrotic after combined treatment (Figure 5).

\section{Discussion}

In the present study, we investigated the radiosensitizing effect of a hypoxic cell radiosensitizer, doranidazole, on C6 intracranial glioma. Doranidazole has a 2-nitroimidazole -based chemical structure with a side chain having low lipophilicity. It is designed to be less neurotoxic due to its BBB-impermeability $[15,16]$. In common with other 


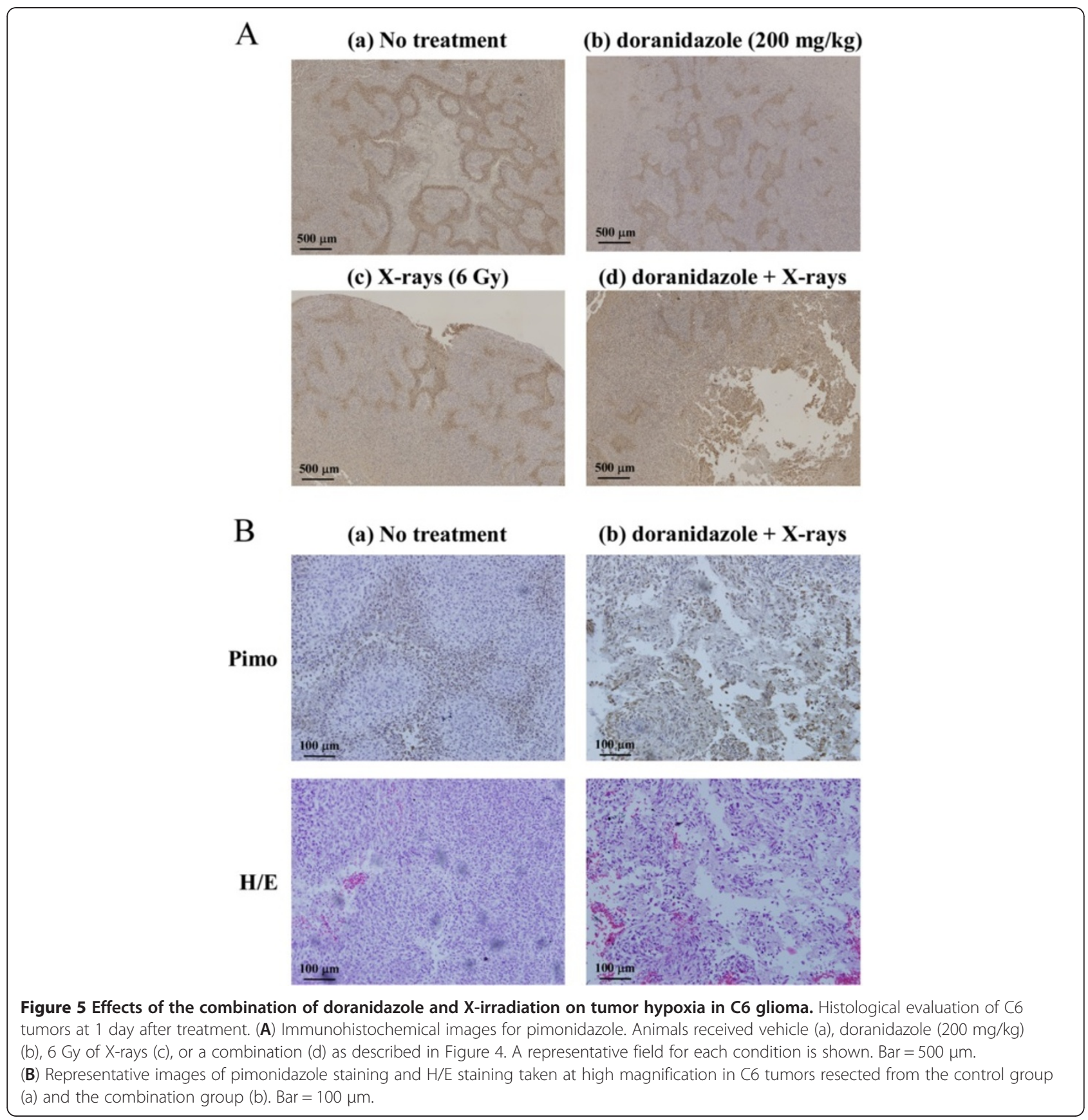

2-nitroimidazole derivatives such as misonidazole and etanidazole, doranidazole is reduced under hypoxic conditions and imported into the cell nucleus, leading to fixation of radiation damage in a manner similar to oxygen [30]. In the present study, it was clearly demonstrated in vitro that doranidazole radiosensitized hypoxic cells as determined by clonogenic survival assay (Figure 1). This radiosensitizing effect was consistent with previous reports $[15,21]$.

Because the delivery of hydrophilic doranidazole into the tumor region is crucial for its radiosensitizing effect, we investigated the extent of the $\mathrm{BBB}$ disruption using Evans blue dye extravasation. Figure 2A clearly shows the penetration of this dye into the tumor region, but not normal brain tissue. The disrupted BBB allows MR-based detection of glioblastoma by extravasation and accumulation of contrast agents such as Gd-DTPA in the interstitial spaces [31]. By using this method, the breakdown of the BBB in C6 glioma was confirmed by CE-MRI with Gd-[DTPA] (Figure 2B and C). Due to its trihydroxyl structure, doranidazole is less lipophilic than misonidazole and etanidazole, with reduced neurotoxicity. The disruption of the $\mathrm{BBB}$ as shown in 
Figure 2 may indicate the feasibility of using doranidazole to treat some intracranial tumors. In fact in the current study, autographic analysis in vivo indicated the obvious accumulation of $\left[{ }^{14} \mathrm{C}\right]$-doranidazole in the tumor region. To our knowledge, our results have clarified for the first time that disruption of the $\mathrm{BBB}$, which has been observed in some types of glioblastoma such as C6 glioma, enabled a lipophobic nitroimidazole analog, doranidazole to be incorporated into the tumor region. To reveal the variability in tumor response to doranidazole based on levels of hypoxia, further investigation using other glioma models will be required.

As mentioned, a number of clinical trials involving a few 2-nitroimidazole-derivatives in combination with radiotherapy have been performed with the objective of improving therapeutic benefit. However, most of them have provided disappointing results with poor enhancement of the efficacy of radiotherapy and severe side effects such as neurotoxicity. To develop an effective therapy with few side effects and sufficient radiosensitizing effects, it is necessary to identify the appropriate tumor type using optimal parameters such as oxygenation status and vascular permeability. Currently, several noninvasive tools are being established for the monitoring of tumor oxygenation and blood perfusion $[32,33]$. To confirm the rationale for using hypoxic cell sensitizers, microenvironmental information on the target tumor should be obtained in preclinical and clinical studies.

\section{Conclusions}

In conclusion, we demonstrated that doranidazole had a radiosensitizing effect on $\mathrm{C} 6$ glioma, a tumor model that shows a wide range of hypoxia and disruption of the BBB. The observation of synergistic tumor growth inhibition by combined treatment with $\mathrm{X}$-irradiation and doranidazole, as shown in Figure 4, clearly indicates the possibility of clinical administration of this drug in the treatment of intracranial glioma. Our study also demonstrated that this radio-sensitization effect was induced through the selective accumulation of doranidazole in a BBB-disrupted tumor. Thus, doranidazole may be a candidate radiosensitizer for use against malignant glioma.

\section{Abbreviations}

BBB: Blood brain barrier; DMEM: Dulbecco's modified Eagle's medium; FBS: Fetal bovine serum; i.v.: intravenous; MSE: Multislice spin-echo; T2Wl: T2weighted image; Gd-DTPA: Gadopentetate dimeglumine; CE-MRI: Contrastenhanced MRl; T1WI: T1-weighted image; H/E: Hematoxylin/eosin; SER: Sensitizing enhancement ratio; ROI: Region of interest.

\section{Competing interests}

NK is an employee of POLA PHARMA INC.; all of the other authors have no competing interests to declare.

\section{Authors' contributions}

$\mathrm{HY}, \mathrm{TA}$ and JK performed the in vitro and in vivo experiments, analyzed the data and prepared the manuscript. TY and SM also participated in the performance of the in vitro experiments. MN prepared the gliomatransplanted animal model. NK synthesized doranidazole and $\left[{ }^{14} \mathrm{C}\right]-$ doranidazole. MK and $\mathrm{OI}$ designed the research and interpreted the data. All authors approved the final version of the manuscript.

\section{Acknowledgements}

This work was supported, in part, by Grants-in-Aid for Basic Scientific Research from the Ministry of Education, Culture, Sports, Science and Technology, Japan (No. 21658106 and No. 21380185 [O.I.], No. 21780267 [T.Y.] and No. 23791375 [H.Y.]), and by the Akiyama Life Science Foundation [H.Y. and T.Y.].

\section{Author details}

'Laboratory of Radiation Biology, Department of Environmental Veterinary Sciences, Graduate School of Veterinary Medicine, Hokkaido University, Kita 18 Nishi 9, Kita-ku, Sapporo, Hokkaido, Japan. 'Laboratory of Veterinary Radiology, Department of Veterinary Sciences, University of Miyazaki, 1-1, Gakuen Kibanadai-nishi, Miyazaki, Miyazaki, Japan. ${ }^{3}$ POLA PHARMA INC, 8-9-5, Nishigotanda, Shinagawa-ku, Tokyo, Japan.

Received: 21 June 2012 Accepted: 3 March 2013

Published: 8 March 2013

\section{References}

1. Burton EC, Prados MD: Malignant gliomas. Curr Treat Options Oncol 2000, 1(5):459-468.

2. Forsyth PA, Cairncross JG: Treatment of malignant glioma in adults. Curr Opin Neurol 1995, 8(6):414-418

3. Robins HI, Chang S, Butowski N, Mehta M: Therapeutic advances for glioblastoma multiforme: current status and future prospects. Curr Oncol Rep 2007, 9(1):66-70.

4. Jensen RL: Brain tumor hypoxia: tumorigenesis, angiogenesis, imaging, pseudoprogression, and as a therapeutic target. J Neurooncol 2009, 92(3):317-335.

5. Chakravarti A, Dicker A, Mehta M: The contribution of epidermal growth factor receptor (EGFR) signaling pathway to radioresistance in human gliomas: a review of preclinical and correlative clinical data. Int J Radiat Oncol Biol Phys 2004, 58(3):927-931.

6. Baumann M, Krause M, Hill R: Exploring the role of cancer stem cells in radioresistance. Nat Rev Cancer 2008, 8(7):545-554.

7. Brown JM, Wilson WR: Exploiting tumour hypoxia in cancer treatment. Nat Rev Cancer 2004, 4(6):437-447.

8. Dewhirst MW: Relationships between cycling hypoxia, HIF-1, angiogenesis and oxidative stress. Radiat Res 2009, 172(6):653-665.

9. Brat DJ, Mapstone TB: Malignant glioma physiology: cellular response to hypoxia and its role in tumor progression. Ann Intern Med 2003, 138(8):659-668.

10. Nagasawa H, Uto Y, Kirk KL, Hori H: Design of hypoxia-targeting drugs as new cancer chemotherapeutics. Biol Pharm Bull 2006, 29(12):2335-2342.

11. Overgaard J: Hypoxic radiosensitization: adored and ignored. $J$ Clin Oncol 2007, 25(26):4066-4074.

12. Kaanders $\mathrm{JH}$, Bussink J, van der Kogel AJ: Clinical studies of hypoxia modification in radiotherapy. Semin Radiat Oncol 2004, 14(3):233-240.

13. Overgaard J, Hansen HS, Andersen AP, Hjelm-Hansen M, Jorgensen $K$, Sandberg E, Berthelsen A, Hammer R, Pedersen M: Misonidazole combined with split-course radiotherapy in the treatment of invasive carcinoma of larynx and pharynx: report from the DAHANCA 2 study. Int J Radiat Oncol Biol Phys 1989, 16(4):1065-1068.

14. Overgaard J, Hansen HS, Overgaard M, Bastholt L, Berthelsen A, Specht L, Lindelov $B$, Jorgensen $K$ : A randomized double-blind phase III study of nimorazole as a hypoxic radiosensitizer of primary radiotherapy in supraglottic larynx and pharynx carcinoma. Results of the Danish Head and Neck Cancer Study (DAHANCA) Protocol 5-85. Radiother Oncol 1998, 46(2):135-146.

15. Oya N, Shibamoto $Y$, Sasai $K$, Shibata T, Murata R, Takagi T, Iwai H, Suzuki T, Abe M: Optical isomers of a new 2-nitroimidazole nucleoside analog (PR-350 series): radiosensitization efficiency and toxicity. Int J Radiat Oncol Biol Phys 1995, 33(1):119-127. 
16. Kuwabara M, lida Y, Inanami O, Sawamura S, Yokoyama K, Tsujitani M: Radiation-chemical properties of the hypoxic cell radiosensitizer doranidazole (PR-350). J Radiat Res (Tokyo) 2002, 43(1):77-88.

17. Aoki M, Furusawa Y, Shibamoto Y, Kobayashi A, Tsujitani M: Effect of a hypoxic cell sensitizer doranidazole on the radiation-induced apoptosis of mouse L5178Y lymphoma cells. J Radiat Res (Tokyo) 2002, 43(2):161-166.

18. Hamasu T, Inanami O, Tsujitani M, Yokoyama K, Takahashi E, Kashiwakura I, Kuwabara M: Post-irradiation hypoxic incubation of X-irradiated MOLT-4 cells reduces apoptotic cell death by changing the intracellular redox state and modulating SAPK/JNK pathways. Apoptosis 2005, 10(3):557-567.

19. Shibamoto Y, Kubota T, Kishii K, Tsujitani M: Radiosensitivity of human pancreatic cancer cells in vitro and in vivo, and the effect of a new hypoxic cell sensitizer, doranidazole. Radiother Oncol 2000, 56(2):265-270.

20. Murata R, Tsujitani M, Horsman MR: Enhanced local tumour control after single or fractionated radiation treatment using the hypoxic cell radiosensitizer doranidazole. Radiother Oncol 2008, 87(3):331-338.

21. Yahiro T, Masui S, Kubota N, Yamada K, Kobayashi A, Kishii K: Effects of hypoxic cell radiosensitizer doranidazole (PR-350) on the radioresponse of murine and human tumor cells in vitro and in vivo. $J$ Radiat Res (Tokyo) 2005, 46(3):363-372.

22. Sunamura M, Karasawa K, Okamoto A, Ogata Y, Nemoto K, Hosotani R, Nishimura Y, Matsui K, Matsuno S: Phase III trial of radiosensitizer PR-350 combined with intraoperative radiotherapy for the treatment of locally advanced pancreatic cancer. Pancreas 2004, 28(3):330-334

23. Deeken JF, Loscher W: The blood-brain barrier and cancer: transporters, treatment, and Trojan horses. Clin Cancer Res 2007, 13(6):1663-1674.

24. Schneider SW, Ludwig T, Tatenhorst L, Braune S, Oberleithner H, Senner V, Paulus W: Glioblastoma cells release factors that disrupt blood-brain barrier features. Acta Neuropathol 2004, 107(3):272-276.

25. Towner RA, Smith N, Doblas S, Garteiser P, Watanabe Y, He T, Saunders D, Herlea O, Silasi-Mansat R, Lupu F: In vivo detection of inducible nitric oxide synthase in rodent gliomas. Free Radic Biol Med 2010, 48(5):691-703.

26. Asanuma T, Doblas S, Tesiram YA, Saunders D, Cranford R, Yasui H, Inanami $\mathrm{O}$, Smith N, Floyd RA, Kotake $Y$, et al: Visualization of the protective ability of a free radical trapping compound against rat C6 and F98 gliomas with diffusion tensor fiber tractography. J Magn Reson Imaging 2008, 28(3):574-587.

27. Liu R, Wen Y, Perez E, Wang X, Day AL, Simpkins JW, Yang SH: 17betaEstradiol attenuates blood-brain barrier disruption induced by cerebral ischemia-reperfusion injury in female rats. Brain Res 2005, 1060(1-2):55-61.

28. Schwartz DL, Powis G, Thitai-Kumar A, He Y, Bankson J, Williams R, Lemos R, Oh J, Volgin A, Soghomonyan S, et al: The selective hypoxia inducible factor-1 inhibitor PX-478 provides in vivo radiosensitization through tumor stromal effects. Mol Cancer Ther 2009, 8(4):947-958.

29. Rapoport SI: Osmotic opening of the blood-brain barrier: principles, mechanism, and therapeutic applications. Cell Mol Neurobiol 2000, 20(2):217-230.

30. Weinmann M, Welz S, Bamberg M: Hypoxic radiosensitizers and hypoxic cytotoxins in radiation oncology. Curr Med Chem Anticancer Agents 2003, 3(5):364-374.

31. Ludemann L, Grieger W, Wurm R, Wust P, Zimmer C: Quantitative measurement of leakage volume and permeability in gliomas, meningiomas and brain metastases with dynamic contrast-enhanced MRI. Magn Reson Imaging 2005, 23(8):833-841.

32. Matsumoto S, Yasui H, Batra S, Kinoshita Y, Bernardo M, Munasinghe JP, Utsumi H, Choudhuri R, Devasahayam N, Subramanian S, et al: Simultaneous imaging of tumor oxygenation and microvascular permeability using Overhauser enhanced MRI. Proc Natl Acad Sci USA 2009, 106(42):17898-17903.

33. Yasui $H$, Matsumoto $S$, Devasahayam N, Munasinghe JP, Choudhuri R, Saito K, Subramanian S, Mitchell JB, Krishna MC: Low-field magnetic resonance imaging to visualize chronic and cycling hypoxia in tumor-bearing mice. Cancer Res 2010, 70(16):6427-6436.

\section{Submit your next manuscript to BioMed Central and take full advantage of:}

- Convenient online submission

- Thorough peer review

- No space constraints or color figure charges

- Immediate publication on acceptance

- Inclusion in PubMed, CAS, Scopus and Google Scholar

- Research which is freely available for redistribution 